\title{
Sex-related differences in the long-term risk of microvascular complications by age at onset of type 1 diabetes
}

\author{
V. Harjutsalo • C. Maric • C. Forsblom • L. Thorn • \\ J. Wadén • P. H. Groop • \\ on behalf of the FinnDiane Study Group
}

Received: 21 January 2011 / Accepted: 8 March 2011 / Published online: 21 April 2011

(C) Springer-Verlag 2011

\begin{abstract}
Aims/hypothesis This study examined sex-related differences in the cumulative risk of proliferative retinopathy (PR) and end-stage renal disease (ESRD) over 40 years of duration of type 1 diabetes according to age at diabetes onset.

Methods We assessed 4,416 patients from the Finnish Diabetic Nephropathy Study population. Kaplan-Meier
\end{abstract}

V. Harjutsalo and C. Maric contributed equally to this study.

Electronic supplementary material The online version of this article (doi:10.1007/s00125-011-2144-2) contains supplementary material, which is available to authorised users.

V. Harjutsalo • C. Forsblom • L. Thorn · J. Wadén · P. H. Groop Folkhälsan Institute of Genetics, Folkhälsan Research Center, Biomedicum Helsinki, University of Helsinki,

Haartmaninkatu 8, P.O. Box 63, 00014 Helsinki, Finland

V. Harjutsalo · C. Forsblom • L. Thorn · J. Wadén · P. H. Groop Division of Nephrology, Department of Medicine,

Helsinki University Central Hospital,

Helsinki, Finland

V. Harjutsalo

National Institute for Health and Welfare,

Diabetes Prevention Unit,

Helsinki, Finland

C. Maric

Department of Physiology and Biophysics, University of Mississippi Medical Center,

Jackson, MS, USA

C. Maric

Women's Health Research Center,

University of Mississippi Medical Center,

Jackson, MS, USA

P. H. Groop $(\bowtie)$

The Baker IDI Heart and Diabetes Institute,

Melbourne, Australia

e-mail: per-henrik.groop@helsinki.fi analysis was used to provide cumulative incidence rates and Cox regression analyses for HRs.

Results There were no sex-related differences in the cumulative incidence of ESRD in patients diagnosed with type 1 diabetes between 0 to 4 and 5 to 9 years. Thereafter the risk started to diverge. The cumulative incidence of ESRD in patients diagnosed between 10 to 14 and $\geq 15$ years was $17.4 \%(95 \%$ CI $13.4-21.2)$ and $13.0 \%(9.6-16.2)$ respectively in women, while in men it was $32.2 \%$ (28.0 $36.1)$ and $24.6 \%(20.8-28.1)$ respectively. The respective HRs were (onset at 10 to 14 years) $1.9(p<0.0001)$ and (onset at $\geq 15$ years) $1.8 \quad(p<0.001)$, respectively. There was no difference in the risk of PR between men and women diagnosed between 0 and 4 years of age, but progressive sex-related differences in the cumulative incidence of PR were observed with increasing age at onset. The HRs for men in the age-at-onset groups 5 to 9 , 10 to 14 and $\geq 15$ years of age were 1.3 (95\% CI $1.0-1.6$ ), 1.3 (1.1-1.6) and $2.1(1.6-2.6)$ compared with women in these groups, respectively.

Conclusions/interpretation The difference between the sexes with regard to risk of diabetic microvascular complications is highly dependent on the age at onset of diabetes. The risk of ESRD and PR risk doubled in men compared with women when age at onset was $\geq 15$ years.

Keywords Age at onset - Diabetic nephropathy - End-stage renal disease (ESRD) · Hormonal changes · Proliferative retinopathy $\cdot$ Sex $\cdot$ Type 1 diabetes

$\begin{array}{ll}\text { Abbreviations } \\ \text { ESRD } & \text { End-stage renal disease } \\ \text { FinnDiane } & \text { Finnish Diabetic Nephropathy Study } \\ \text { PR } & \text { Proliferative retinopathy } \\ \text { SHBG } & \text { Sex-hormone-binding globulin }\end{array}$




\section{Introduction}

Most previous studies suggest that male sex increases the risk of microvascular complications associated with type 1 diabetes, especially that of diabetic nephropathy. In adult men, development and progression of albuminuria is greater than in age-matched women with type 1 diabetes [1, 2]; however, the impact of different ages at onset of diabetes was not taken into account in these studies. Some data suggest that, in addition to sex, the age at onset of type 1 diabetes is also an important determinant of the overall risk of developing diabetic microvascular complications [3, 4]. But the question of whether age at onset of diabetes has a similar impact on the risk of microvascular complications in men as in women is inadequately studied. Moreover, most previous studies are related to diabetic nephropathy or microalbuminuria, and have found that male sex is a risk factor $[5,6]$. However, studies on sex or age at onset in relation to diabetic retinopathy are sparse and controversial [7-9]. Finally, very few studies have investigated the interaction between sex and age at onset with regard to any microvascular complication $[3,4]$.

Several studies have shown that diabetic microvascular complications, specifically, retinopathy and diabetic nephropathy, rarely develop before puberty $[3,4,10-13]$. While the relatively short period of time between diagnosis of type 1 diabetes and onset of puberty may explain why complications rarely develop before puberty, it is likely that more physiological reasons exist for this observation. Interestingly, the onset of puberty itself greatly accelerates the development of microvascular disease associated with retinopathy $[14,15]$ and diabetic nephropathy $[15,16]$ in type 1 diabetes patients. Poor glycaemic control during puberty, which is related to poor compliance with diet and insulin dosage, is thought to contribute to the risk of long-term microvascular complications [17]. However, other endocrine changes during puberty, such as growth and sexual maturation, may also influence the development and progression of end-organ complications that are associated with type 1 diabetes later in life.

Boys and girls reach puberty at different ages and undergo different biological changes during their sexual maturation [18]. It is therefore conceivable that long-term microvascular complications, the risk of which is accelerated by puberty, may be sex-dependent. Studies that have examined the impact of sex on the development and progression of diabetic end-organ complications, as well as on type 1 diabetes as such, have reported conflicting findings. In most populations studied, the incidence of type 1 diabetes was similar in girls and boys, increasing with age and peaking at puberty in both sexes [19-21]. After puberty, however, the incidence of type 1 diabetes remains high in young adult men, but decreases in young women $[20,22,23]$. Thus, the aim of the present study was to examine the long-term impact of sex on the risk of developing proliferative retinopathy (PR) and end-stage renal disease (ESRD) in adulthood according to age at onset of type 1 diabetes.

\section{Methods}

Data collection The data for the analyses presented are derived from the Finnish Diabetic Nephropathy Study (FinnDiane). FinnDiane is a nationwide, comprehensive multicentre study, designed to identify genetic and environmental risk factors for diabetic complications, with special emphasis on diabetic nephropathy in patients with type 1 diabetes. A detailed description of the FinnDiane study recruitment protocol has been previously described [24]. In brief, eligible patients recruited into the study were those with onset of type 1 diabetes at the age of 40 years or less and in whom insulin treatment was initiated within 1 year of diagnosis. An additional criterion for selection was C-peptide levels $\leq 0.3 \mathrm{nmol} / \mathrm{l}$. For a list of physicians and nurses at each of the FinnDiane centres participating in patient recruitment and characterisation, see electronic supplementary material (ESM).

At baseline patients underwent a thorough clinical examination that took place in conjunction with a regular visit to the attending physician. Details of clinical characteristics of patients, including age at diagnosis of diabetes, PR and ESRD, and laboratory measurements were obtained from medical files by the attending physician using a standardised questionnaire. Follow-up started at the time of diagnosis of diabetes and ended at the time of diagnosis of PR or ESRD. Patients without these complications contributed to follow-up until their last follow-up visit, the latest screening of their medical files or until their death.

The ethics committees of all participating centres approved the study protocol. Written informed consent was obtained from each patient and the study was performed in accordance with the Declaration of Helsinki as revised in the year 2000.

Definition of $P R$ and ESRD Finland has a nationally organised diabetes care system and according to the national management guidelines, all patients with type 1 diabetes of 5 years duration and longer are screened for retinopathy approximately every 2 nd year. The screenings are performed by ophthalmoscopy followed by fundus photography, based on which candidates for laser treatment are selected. In this study, laser treatment was considered as evidence of PR. Although laser treatment is also used for clinically significant macular oedema, most patients receiving laser treatment in this study received it for PR. In a separate subset of the FinnDiane patient population we saw that most of those patients receiving laser treatment for macular oedema later also received laser treatment for PR. ESRD 
Table 1 Number of men and women diagnosed with PR and ESRD according to the age at onset of type 1 diabetes

\begin{tabular}{|c|c|c|c|c|c|c|c|c|}
\hline \multirow[t]{2}{*}{ Group $^{\text {a }}$} & \multicolumn{3}{|l|}{ PR analysis } & \multicolumn{3}{|c|}{ ESRD analysis } & \multicolumn{2}{|c|}{ Duration of diabetes (years) } \\
\hline & Patients, $n$ & Patients, $n(\%)$ & Person-years & Patients, $n$ & Patients, $n(\%)$ & Person-years & PR, median (IQR) & ESRD, median (IQR) \\
\hline All groups & 3,828 & 1,232 & 77,924 & 4,416 & 569 & 125,991 & $21.0(11.6-30.4)$ & $29.5(19.5-38.5)$ \\
\hline \multicolumn{9}{|l|}{$0-4$ years } \\
\hline All & 428 & $213(49.8)$ & 11,155 & 492 & $83(16.9)$ & 17,781 & $28.6(21.0-36.7)$ & $37.5(29.5-44.5)$ \\
\hline Men & 218 & $111(50.9)$ & 5,635 & 249 & $41(16.5)$ & 9,017 & $28.3(20.8-36.4)$ & $37.5(29.5-44.5)$ \\
\hline Women & 210 & $102(48.6)$ & 5,519 & 243 & $42(17.3)$ & 8,764 & $28.3(20.8-36.4)$ & $37.5(30.5-44.5)$ \\
\hline \multicolumn{9}{|l|}{$5-9$ years } \\
\hline All & 803 & $359(44.7)$ & 18,089 & 909 & $164(18.0)$ & 29,400 & $24.2(16.4-33.2)$ & $33.6(25.5-41.5)$ \\
\hline Men & 342 & $164(48.0)$ & 7,442 & 401 & $74(18.5)$ & 13,036 & $23.8(15.3-33.0)$ & $33.5(25.5-41.5)$ \\
\hline Women & 461 & $195(42.3)$ & 10,647 & 508 & $90(17.7)$ & 16,364 & $24.9(17.3-33.2)$ & $34.5(25.5-43.5)$ \\
\hline \multicolumn{9}{|l|}{ 10-14 years } \\
\hline All & 935 & $325(34.8)$ & 19,745 & 1,024 & $153(14.9)$ & 30,617 & $22.7(13.3-31.7)$ & $31.5(22.5-40.5)$ \\
\hline Men & 462 & $176(38.1)$ & 9,453 & 520 & $98(18.9)$ & 15,192 & $21.8(13.1-31.5)$ & $31.5(22.0-40.3)$ \\
\hline Women & 473 & 149 (31.5) & 10,292 & 504 & 55 (10.9) & 15,425 & $23.1(13.6-32.0)$ & $31.5(22.5-40.5)$ \\
\hline \multicolumn{9}{|l|}{$\geq 15$ years } \\
\hline All & 1,662 & $335(20.2)$ & 28,936 & 1,991 & $169(8.0)$ & 48,193 & $15.5(7.3-25.1)$ & $24.5(15.5-33.5)$ \\
\hline Men & 919 & $226(24.6)$ & 15,161 & 1,117 & $118(10.0)$ & 26,831 & $14.7(6.4-25.2)$ & $24.3(14.5-34.5)$ \\
\hline Women & 743 & 109 (14.7) & 13,775 & 874 & $51(5.5)$ & 21,362 & $16.0(7.9-24.9)$ & $24.5(16.5-33.5)$ \\
\hline
\end{tabular}

IQR, interquartile range

${ }^{\text {a }}$ By age at onset

was defined as dialysis treatment or having received a kidney transplant. The analysis for PR included 3,828 patients, that for ESRD 4,416 patients.

Statistical methods For the analysis, the patients were divided into four groups according to the age at onset of diabetes, i.e.: $0-4,5-9,10-14$ and $\geq 15$ years of age. Sexrelated differences in the risk of microvascular complications were studied within each age-at-onset group. The cumulative incidence of PR and ESRD was estimated using the KaplanMeier method. Deaths without event were treated as censored observations. Analyses were conducted stratifying the data according to the age at onset of diabetes. Differences between the groups were tested using the logrank test. The HRs were estimated using Cox proportional hazards modelling. Interaction between sex and age at onset was tested. Because patients with ESRD frequently have PR, analyses of risk of PR were also conducted excluding patients with ESRD. All analyses were performed using a software package (SAS 9.2; SAS Institute, Cary, NC, USA).

\section{Results}

Patient characteristics The number of patients analysed in the study is shown in Table 1. Of the total number of patients included, 1,232 developed PR and 569 developed ESRD. The median duration (interquartile range) of followup was 21.0 (11.6-30.4) years in the PR analysis and 29.5 (19.5-38.5) years in the ESRD analysis. Interaction between sex and age at onset of diabetes was significant for $\operatorname{PR}(p=0.006)$ and $\operatorname{ESRD}(p=0.004)$, indicating that the effect of sex on the risk of PR and ESRD depends on age at onset.

Sex-related differences in the cumulative risk of ESRD No sex-related differences in the cumulative risk of ESRD over 40 years of diabetes duration was evident in patients diagnosed with diabetes between 0 to 4 (Fig. 1a) and 5 to 9 years of age (Fig. 1b). Similarly to PR, sex-related differences in the long-term risk of ESRD increased with increasing age at onset of type 1 diabetes; however, differences were only apparent when age at onset was 10 years or more (Fig. 1c, d and Table 2). The cumulative risk of ESRD in patients diagnosed between 10 and 14 years of age was $17.4 \%$ (95\% CI 13.4-21.2) in women and $32.2 \%$ (95\% CI 28.0-36.1) in men, HR $1.91(p<0.0001$; Table 2). If onset of diabetes was at $\geq 15$ years of age, the cumulative risk of ESRD was $13.0 \%$ (95\% CI 9.6-16.2) in women and $24.6 \%$ (95\% CI 20.8-28.1) in men, HR 1.81 $(p<0.001)$. Thus, sex-related differences in the long-term risk of ESRD were apparent when age at onset of type 1 diabetes was 10 years or more, with risk in men being twice 
that in women. Interestingly, the chronological age at which the risk curves started to diverge based on sex was 32 to 37 years, regardless of the age at onset of diabetes. Similarly to PR, the overall risk of ESRD was $36 \%$ ( $p=0.0003)$ higher in men than in women, suggesting that male sex is a risk factor for ESRD as well as PR.

Interestingly, if all age-at-onset groups were pooled, sexrelated differences were not noticeable over 30 years of duration of diabetes. The 30 year cumulative risk of ESRD was $9.6 \%(95 \%$ CI $8.1-11.0)$ in women and $11.5 \%(95 \%$ CI $10.0-12.9)$ in men, $\mathrm{HR}=1.23(p=0.07)$.

Sex-related differences in the cumulative risk of $P R$ No difference in the risk of PR was observed between men and women diagnosed with type 1 diabetes between 0 and 4 years of age (Fig. 2a). However, sex-related differences in the cumulative risk of PR over 40 years of diabetes did become apparent with increasing age at onset of diabetes (Fig. 2b-d). The corresponding HRs for men in the age-atonset groups 5 to 9 years, 10 to 14 years and $\geq 15$ years of age were 1.26 (95\% CI 1.03-1.55), 1.32 (95\% CI $1.06-$ 1.64 ) and 2.06 (95\% CI 1.64-2.58), respectively (Table 2). As with ESRD, the patient age at which sex-related differences were observed was between 35 and 37 years, irrespective of age at onset of diabetes. The overall risk of PR was $39 \%(p<0.0001)$ higher in men than in women, suggesting that male sex is a risk factor for PR. The 40 year cumulative risk of PR was $72.0 \%$ (95\% CI 70.9-73.0) in men and $52.6 \%$ (95\% CI 50.6-54.4) in women.

When data analysed excluded patients who also had ESRD, the sex-related difference remained similar (Table 2). In fact, in the oldest age-at-onset group, the sex-related difference was even strengthened.

\section{Discussion}

This study shows that the risk of diabetic microvascular complications, in the form of PR and ESRD, over 40 years of type 1 diabetes duration is highly dependent on age at onset of diabetes and on sex. Interestingly, when all age-atonset groups were pooled, the sex-related difference in the risk of ESRD was not noticeable at 30 years of disease duration, only becoming noticeable at 40 years of duration. In essence, only stratification by age at onset
Fig. 1 Sex-related differences in the cumulative risk of ESRD over 40 years of type 1 diabetes according to age of diabetes onset: (a) 0 to 4 years, (b) 5 to 9 years, (c) 10 to 14 years and $(\mathbf{d}) \geq 15$ years. F, females; $\mathrm{M}$, males
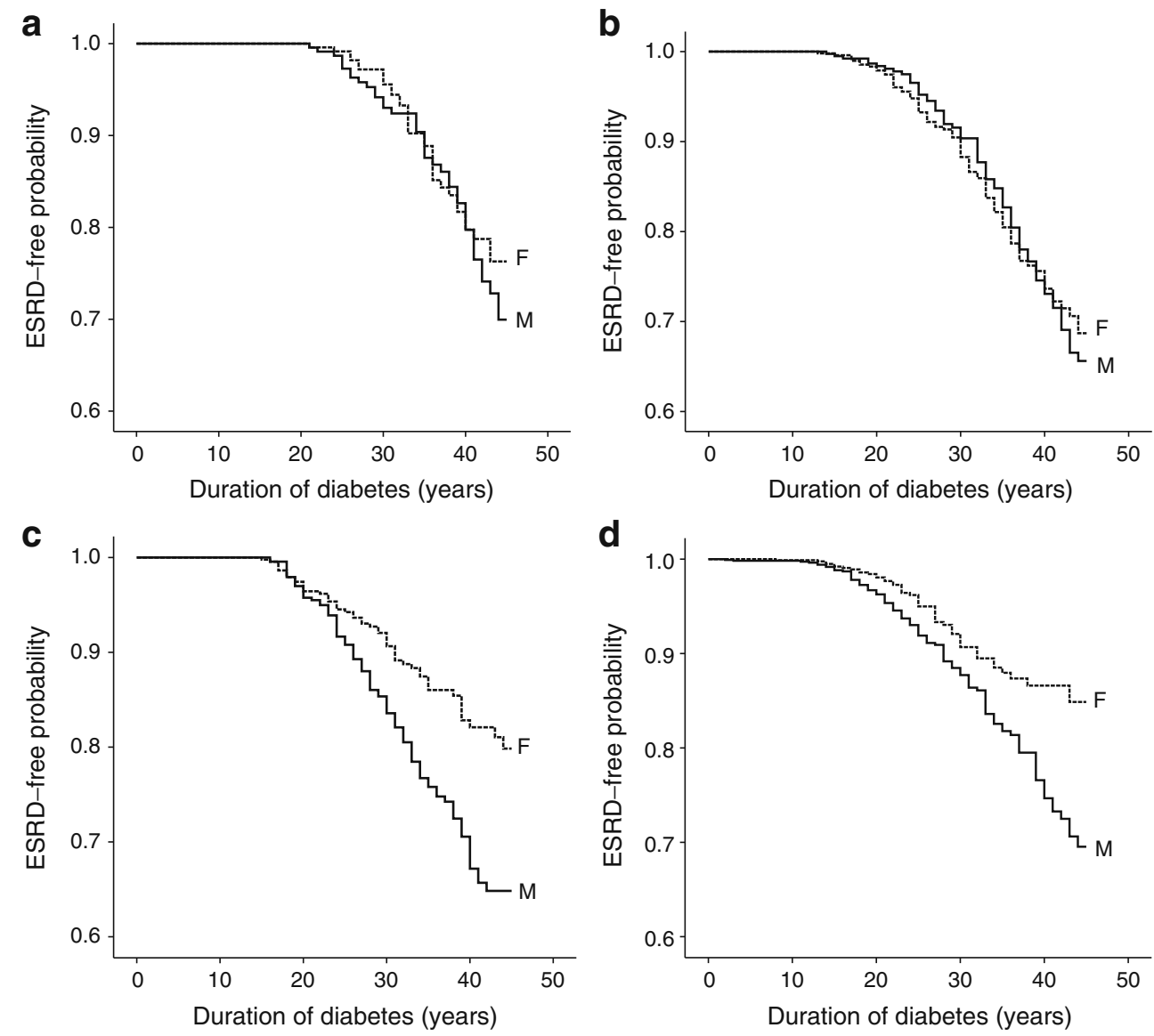
Table 2 Sex-related differences in the 40 year cumulative risk of PR and ESRD according to age of diagnosis of type 1 diabetes

\begin{tabular}{|c|c|c|c|c|c|c|c|c|c|c|c|c|}
\hline \multirow[t]{2}{*}{ Group $^{a}$} & \multicolumn{4}{|c|}{ PR analysis } & \multicolumn{4}{|c|}{ PR analysis with ESRD patients excluded } & \multicolumn{4}{|c|}{ ESRD analysis } \\
\hline & $\mathrm{CR}^{\mathrm{b}}$ & HR & $95 \% \mathrm{CI}$ for $\mathrm{HR}$ & $p$ value & $\mathrm{CR}^{\mathrm{b}}$ & $\mathrm{HR}$ & $95 \% \mathrm{CI}$ for $\mathrm{HR}$ & $p$ value & $\mathrm{CR}^{\mathrm{b}}$ & $\mathrm{HR}$ & $95 \% \mathrm{CI}$ for $\mathrm{HR}$ & $p$ value \\
\hline All & 62.2 & & & & 55.8 & & & & 23.0 & & & \\
\hline \multicolumn{13}{|l|}{$0-4$ years } \\
\hline Men & 72.1 & 1.08 & $0.82-1.41$ & 0.58 & 61.5 & 1.09 & $0.79-1.49$ & 0.61 & 19.8 & 0.95 & $0.61-1.46$ & 0.81 \\
\hline Women & 68.2 & 1.00 & - & & 61.7 & 1.00 & - & & 19.8 & 1.00 & - & \\
\hline \multicolumn{13}{|l|}{$5-9$ years } \\
\hline Men & 73.6 & 1.26 & $1.03-1.55$ & 0.03 & 70.8 & 1.40 & $1.09-1.78$ & 0.008 & 26.3 & 1.01 & $0.74-1.37$ & 0.97 \\
\hline Women & 60.1 & 1.00 & - & & 54.1 & 1.00 & & & 25.7 & 1.00 & - & \\
\hline \multicolumn{13}{|c|}{$10-14$ years } \\
\hline Men & 69.6 & 1.32 & $1.06-1.64$ & 0.01 & 63.1 & 1.36 & $1.04-1.77$ & 0.02 & 32.2 & 1.91 & $1.37-2.66$ & $<0.0001$ \\
\hline Women & 49.7 & 1.00 & - & & 42.7 & 1.00 & & - & & 17.4 & 1.00 & - \\
\hline \multicolumn{13}{|l|}{$\geq 15$ years } \\
\hline Men & 72.6 & 2.06 & $1.64-2.58$ & $<0.0001$ & 67.6 & 2.46 & $1.87-3.25$ & $<0.0001$ & 24.6 & 1.81 & $1.30-2.51$ & $<0.0001$ \\
\hline Women & 35.9 & 1.00 & - & & 30.4 & 1.00 & & - & & 13.0 & 1.00 & - \\
\hline
\end{tabular}

Overall, men had 39\% (95\% CI 1.24-1.56 for HR, $p<0.0001)$ higher risk of PR and 36\% (1.15-1.61 for HR, $p=0.0003)$ higher risk of ESRD than women

${ }^{\mathrm{a}}$ By age at onset; ${ }^{\mathrm{b}}$ cumulative risk

Fig. 2 Sex-related differences in the cumulative risk of PR over 40 years of type 1 diabetes according to age of diabetes onset: (a) 0 to 4 years, (b) 5 to 9 years, (c) 10 to 14 years and $(\mathbf{d}) \geq 15$ years. F, females; $\mathrm{M}$, males
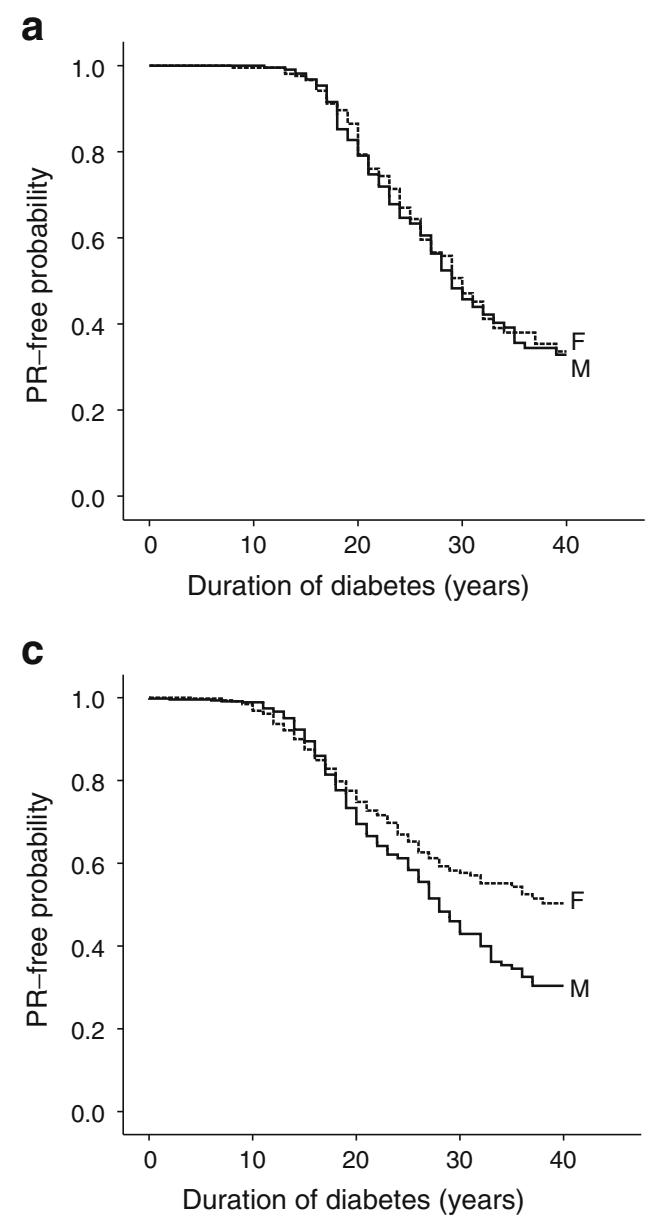
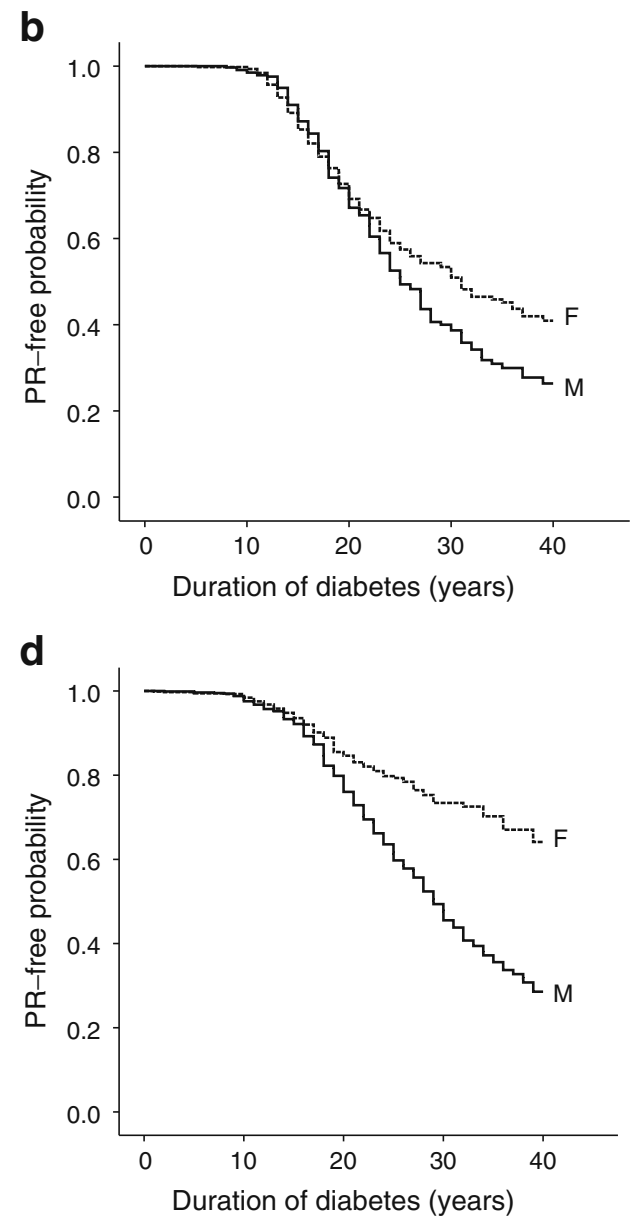
and a long enough follow-up time revealed the sexrelated difference. This sex-related divergence in ESRD was evident at age at onset of 10 years and older; in PR, it was evident when age at onset was between 5 and 9 years, and increased thereafter.

While male sex is thought to be a risk factor for development of diabetic microvascular complications $[1,2]$, the majority of epidemiological studies commonly group women and men together in the analyses, thus potentially masking the impact of sex on disease development and progression. Our study shows the importance of considering age at onset of diabetes revealing sex-related differences. Specifically, this study shows that sex-related differences in the long-term risk of PR first became evident in patients diagnosed with type 1 diabetes at the age of 5 to 9 years, becoming more pronounced with increasing age at disease onset. In the case of ESRD, sex-related differences were not evident in patients diagnosed before 10 years of age, but did emerge in patients with pubertal and adult onset of type 1 diabetes. These observations suggest that the critical period of sexual maturation and permanent hormonal changes are permissive to development of longterm complications, this being more true of men than women. We do not believe that these differences are related to differences in glycaemic control, as the mean $\mathrm{HbA}_{1 \mathrm{c}}$ value based on one cross-sectional measurement was similar in men and women, i.e. $8.38 \%$ vs $8.45 \%$ (data not shown).

Findings from the present study support previous reports that the male sex is a risk factor for ESRD $[1,2]$. However, the impact of sex on the risk of retinopathy is rarely reported and the available data are conflicting [25, 26]. Some studies have reported that men have a greater risk of diabetic retinopathy, but not PR $[9,25]$. Our study shows that male sex is a risk factor for PR and that this risk is stronger with increasing age at onset of diabetes.

In agreement with our study, a recent nationwide population-based Swedish study found no sex-related difference in the risk of ESRD in patients diagnosed under age of 10 years [27]. This study also failed to find a significant difference between sexes when age at onset was between 10 and 19 years. However, thereafter sex-related differences did emerge. It should be noted that that study reported cumulative incidence only over 30 years of diabetes, while our study shows that sex-related differences do not become noticeable until 40 years of diabetes duration. Thus, a longer duration of diabetes appears to be necessary for ESRD to develop in many predisposed patients. Taken together, these observations underscore the impact of sex in the risk of ESRD.

One mechanism underlying the sex-related difference in the development of microvascular complications may be epigenetic modifications. It is known that sex hormones and high glucose trigger epigenetic modifications, thus regulating gene expression without changing DNA sequences $[28,29]$. Changes in the hormonal profile before and after puberty, and prior to or after diagnosis of diabetes, may also differentially trigger epigenetic modifications. It is therefore conceivable that differential timing and order of factors causing epigenetic modifications may play a key role in determining the risk of microvascular complications in men and women. Bearing this in mind, sex-related differences in the development of microvascular complications according to age at onset may be explained as follows. First, when men and women have age at onset below 5 years, no differences emerge regarding lifetime risk of PR and ESRD. Thus, exposure to hyperglycaemia prior to hormonal changes equally modifies predisposing genes in both sexes, leading to a similar risk pattern in both sexes. Second, when diabetes starts at the age of 5 to 9 years, it coincides with pre-pubertal changes. Simultaneous occurrence of diabetes and these pre-pubertal hormonal changes may thus lead to different risk profiles in men and women. Third, hormonal changes during puberty leading to sexual maturation may further differentiate the risk profiles in men and women. Finally, if pre-pubertal and pubertal hormonal changes occur before onset of type 1 diabetes and glycaemic exposure, this seems to favour women.

Our observations suggest that the much discussed pubertal onset of diabetes as a risk factor for long-term diabetic organ complications is specific to men, but that early age of development of diabetes poses similar risk for men and women. Also in post-pubertal onset of type 1 diabetes, the sex-related difference remains, but favours women. Puberty is characterised by several hormonal and metabolic changes, including the acceleration and cessation of somatic growth and onset of reproductive capacity. Our study suggests that these changes appear to permit the development of organ complications later in life, particularly in men. Hormones involved in the control of somatic growth and reproduction during puberty, including growth hormone and insulin-like growth factor, have already been implicated in the development of diabetic microvascular complications [30].

Levels of testosterone and sex-hormone-binding globulin (SHBG) have been linked to the risk of diabetic nephropathy and diabetic retinopathy. It has consistently been found that testosterone and SHBG have an effect, but the direction of the effect seems to vary from study to study. In some studies reduced levels of testosterone, while in other studies increased levels of testosterone, were found to correlate with decline in microalbuminuria or diabetic nephropathy $[31,32]$. In the Wisconsin Epidemiologic Study of Diabetic Retinopathy participants who progressed to PR or preproliferative retinopathy had lower levels of serum SHBG than controls [33]. Moreover, serum testosterone 
concentrations were significantly higher in male diabetic participants with PR [34]. Chaurasia et al. also found higher testosterone levels in patients with proliferative diabetic retinopathy [35].

The fact that women appear to have a lower overall cumulative risk of complications than men suggests that female sex is a protective factor against the development of microvascular complications. Several experimental studies have demonstrated a renoprotective role of exogenous oestrogens in models of type 1 diabetic retinopathy and nephropathy [36-39]. However, an experimental model of type 1 diabetes has also, however, shown that relative balance, rather than the absolute levels of sex hormones, correlates with conditions associated with diabetic complications [40].

Interestingly, the attained chronological age at which the sex-related differences in the risk of both ESRD and PR first become apparent was around 32 to 37 years. It is, of course, possible that changes developing around the age of 32 to 37 years are not entirely related to diabetes, but rather biological changes that are specific to men at this time in life.

To our knowledge, this is the first study to report sexrelated differences in the 40 year cumulative risk of PR and ESRD based on age at onset of type 1 diabetes. One of its major strengths is the large number of patients included in the analysis and their long-term (40 years) follow-up. The main limitation is that FinnDiane is not, by strict definition, a population-based study, although it is a large nationwide study. Thus, patients who died before recruitment never reached this cohort, leading to selection of surviving patients only. However, confirmation of our results comes from a population-based study from Sweden [27], and we found the same pattern with longer follow-up time.

In summary, our study suggests that the difference between the sexes with regard to long-term risk of developing diabetic microvascular complications is highly dependent on the age at onset of type 1 diabetes. These data suggest that, in addition to the classical risk factors for diabetic retinopathy and nephropathy, sex-specific initiation of diabetes interventions may be necessary for effective prevention from complications in these patients.

\footnotetext{
Acknowledgements The authors would like to acknowledge all of the physicians and nurses at each of the FinnDiane centres participating in patient recruitment and characterisation (see ESM). This research was supported by grants from the Folkhälsan Research Foundation, Wilhelm and Else Stockmann Foundation, Liv och Hälsa Foundation and The Finnish Medical Society (to P.-H. Groop), and the Research Career Enhancement Award from the American Physiological Society (to C. Maric).
}

Duality of interest The authors declare that there is no duality of interest associated with this manuscript.

\section{References}

1. Andersen AR, Christiansen JS, Andersen JK, Kreiner S, Deckert T (1983) Diabetic nephropathy in type 1 (insulin-dependent) diabetes: an epidemiological study. Diabetologia 25:496-501

2. Jacobsen P, Rossing K, Tarnow L et al (1999) Progression of diabetic nephropathy in normotensive type 1 diabetic patients. Kidney Int (Suppl) 71:S101-S105

3. Schultz CJ, Konopelska-Bahu T, Dalton RN et al (1999) Microalbuminuria prevalence varies with age, sex, and puberty in children with type 1 diabetes followed from diagnosis in a longitudinal study. Oxford Regional Prospective Study Group. Diabetes Care 22:495-502

4. Svensson M, Nyström L, Schon S, Dahlquist G (2006) Age at onset of childhood-onset type 1 diabetes and the development of end-stage renal disease: a nationwide population-based study. Diabetes Care 29:538-542

5. Raile K, Galler A, Hofer S et al (2007) Diabetic nephropathy in 27,805 children, adolescents, and adults with type 1 diabetes: effect of diabetes duration, A1C, hypertension, dyslipidemia, diabetes onset, and sex. Diabetes Care 30:2523-2528

6. Harjutsalo V, Katoh S, Sarti C, Tajima N, Tuomilehto J (2004) Population-based assessment of familial clustering of diabetic nephropathy in type 1 diabetes. Diabetes 53:2449-2454

7. Klein R, Knudtson MD, Lee KE, Gangnon R, Klein BE (2008) The Wisconsin Epidemiologic Study of Diabetic Retinopathy: XXII. The twenty-five-year progression of retinopathy in persons with type 1 diabetes. Ophthalmology 115:1859-1868

8. Donaghue KC, Fairchild JM, Craig ME et al (2003) Do all prepubertal years of diabetes duration contribute equally to diabetes complications? Diabetes Care 26:1224-1229

9. Hietala K, Harjutsalo V, Forsblom C, Summanen P, Groop PH (2010) Age at onset and the risk of proliferative retinopathy in type 1 diabetes. Diabetes Care 33:1315-1319

10. Rudberg S, Ullman E, Dahlquist G (1993) Relationship between early metabolic control and the development of microalbuminuria - a longitudinal study in children with type 1 (insulin-dependent) diabetes mellitus. Diabetologia 36:13091314

11. Mauer M, Drummond K (2002) The early natural history of nephropathy in type 1 diabetes: I. Study design and baseline characteristics of the study participants. Diabetes 51:15721579

12. Drummond KN, Kramer MS, Suissa S et al (2003) Effects of duration and age at onset of type 1 diabetes on preclinical manifestations of nephropathy. Diabetes 52:1818-1824

13. Janner M, Knill SE, Diem P, Zuppinger KA, Mullis PE (1994) Persistent microalbuminuria in adolescents with type I (insulindependent) diabetes mellitus is associated to early rather than late puberty. Results of a prospective longitudinal study. Eur J Pediatr 153:403-408

14. Klein R, Klein BE, Moss SE, Cruickshanks KJ (1998) The Wisconsin epidemiologic study of diabetic retinopathy: XVII. The 14-year incidence and progression of diabetic retinopathy and associated risk factors in type 1 diabetes. Ophthalmology 105:1801-1815

15. Kostraba JN, Dorman JS, Orchard TJ et al (1989) Contribution of diabetes duration before puberty to development of microvascular complications in IDDM subjects. Diabetes Care 12:686-693

16. Lawson ML, Sochett EB, Chait PG, Balfe JW, Daneman D (1996) Effect of puberty on markers of glomerular hypertrophy and hypertension in IDDM. Diabetes 45:51-55

17. The Epidemiology of Diabetes Intervention and Complications (EDIC) Study (2003) Sustained effect of intensive treatment of type 1 diabetes mellitus on development and progression of 
diabetic nephropathy: the Epidemiology of Diabetes Interventions and Complications (EDIC) Study. JAMA 290:2159-2167

18. Kauffman AS (2010) Coming of age in the kisspeptin era: sex differences, development, and puberty. Mol Cell Endocrinol 324:51-63

19. Harjutsalo V, Sjoberg L, Tuomilehto J (2008) Time trends in the incidence of type 1 diabetes in Finnish children: a cohort study. Lancet 371:1777-1782

20. Soltesz G, Patterson CC, Dahlquist G (2007) Worldwide childhood type 1 diabetes incidence-what can we learn from epidemiology? Pediatr Diabetes 8(Suppl 6):6-14

21. Zhang H, Xia W, Yu Q et al (2008) Increasing incidence of type 1 diabetes in children aged 0-14 years in Harbin, China (1990 2000). Prim Care Diabetes 2:121-126

22. Lammi N, Taskinen O, Moltchanova E et al (2007) A high incidence of type 1 diabetes and an alarming increase in the incidence of type 2 diabetes among young adults in Finland between 1992 and 1996. Diabetologia 50:1393-1400

23. Ostman J, Lonnberg G, Arnqvist HJ et al (2008) Gender differences and temporal variation in the incidence of type 1 diabetes: results of 8012 cases in the nationwide Diabetes Incidence Study in Sweden 1983-2002. J Intern Med 263:386394

24. Thorn LM, Forsblom C, Fagerudd J et al (2005) Metabolic syndrome in type 1 diabetes: association with diabetic nephropathy and glycemic control (the FinnDiane Study). Diabetes Care 28:2019-2024

25. Monti MC, Lonsdale JT, Montomoli C, Montross R, Schlag E, Greenberg DA (2007) Familial risk factors for microvascular complications and differential male-female risk in a large cohort of American families with type 1 diabetes. J Clin Endocrinol Metab 92:4650-4655

26. Harvey JN, Allagoa B (2004) The long-term renal and retinal outcome of childhood-onset type 1 diabetes. Diabet Med 21:26-31

27. Mollsten A, Svensson M, Waernbaum I et al (2010) Cumulative risk, age at onset, and sex-specific differences for developing endstage renal disease in young patients with type 1 diabetes: a nationwide population-based cohort study. Diabetes 59:18031808

28. El-Osta A, Brasacchio D, Yao D et al (2008) Transient high glucose causes persistent epigenetic changes and altered gene expression during subsequent normoglycemia. J Exp Med 205:2409-2417

29. Kaminsky Z, Wang SC, Petronis A (2006) Complex disease, gender and epigenetics. Ann Med 38:530-544

30. Cummings EA, Sochett EB, Dekker MG, Lawson ML, Daneman D (1998) Contribution of growth hormone and IGF-I to early diabetic nephropathy in type 1 diabetes. Diabetes 47:1341-1346

31. Amin R, Schultz C, Ong K et al (2003) Low IGF-I and elevated testosterone during puberty in subjects with type 1 diabetes developing microalbuminuria in comparison to normoalbuminuric control subjects: the Oxford Regional Prospective Study. Diabetes Care 26:1456-1461

32. Maric C, Forsblom C, Thorn L, Waden J, Groop PH (2010) Association between testosterone, estradiol and sex hormone binding globulin levels in men with type 1 diabetes with nephropathy. Steroids 75:772-778

33. Haffner SM, Klein R, Moss SE, Klein BE (1993) Sex hormones and the incidence of severe retinopathy in male subjects with type I diabetes. Ophthalmology 100:1782-1786

34. Haffner SM, Klein R, Dunn JF, Moss SE, Klein BE (1990) Increased testosterone in type I diabetic subjects with severe retinopathy. Ophthalmology 97:1270-1274

35. Chaurasia RK, Singh R, Agrawal JK, Maurya OP (1993) Sex hormones and diabetic retinopathy. Ann Ophthalmol 25:227-230

36. Mankhey RW, Bhatti F, Maric C (2005) 17beta-Estradiol replacement improves renal function and pathology associated with diabetic nephropathy. Am J Physiol Renal Physiol 288: F399-F405

37. Keck M, Romero-Aleshire MJ, Cai Q, Hoyer PB, Brooks HL (2007) Hormonal status affects the progression of STZ-induced diabetes and diabetic renal damage in the VCD mouse model of menopause. Am J Physiol Renal Physiol 293:F193-F199

38. Kaja S, Yang SH, Wei J et al (2003) Estrogen protects the inner retina from apoptosis and ischemia-induced loss of Vesl-1L/ Homer 1c immunoreactive synaptic connections. Invest Ophthalmol Vis Sci 44:3155-3162

39. Maric C, Sullivan S (2008) Estrogens and the diabetic kidney. Gend Med 5(Suppl A):S103-S113

40. Xu Q, Wells CC, Garman JH, Asico L, Escano CS, Maric C (2008) Imbalance in sex hormone levels exacerbates diabetic renal disease. Hypertension 51:1218-1224 